\title{
A few cosmological implications of tensor nonlocalities
}

\author{
Pedro G. Ferreira ${ }^{1, *}$ and Antonio L. Maroto ${ }^{2, \dagger}$ \\ ${ }^{1}$ Astrophysics, University of Oxford, DWB, Keble Road, Oxford OX1 3RH, United Kingdom \\ ${ }^{2}$ Departamento de Física Teórica, Universidad Complutense de Madrid, 28040 Madrid, Spain
}

(Received 4 October 2013; published 2 December 2013)

\begin{abstract}
We consider nonlocal gravity theories that include tensor nonlocalities. We show that in the cosmological context, the tensor nonlocalities, unlike scalar ones, generically give rise to growing modes. An explicit example with quadratic curvature terms is studied in detail. Possible consequences for recent nonlocal cosmological models proposed in the literature are also discussed.
\end{abstract}

DOI: 10.1103/PhysRevD.88.123502

PACS numbers: 98.80.-k, 95.36.+x, 98.80.Cq

\section{INTRODUCTION}

The possibility that general relativity (GR) may be modified on different length scales is an area of active research [1]. The overwhelming majority of theories proposed to replace GR involve local functions of the degrees of freedom. Some of the most popular proposals work purely with the metric tensor, constructing functions of the curvature tensors and scalars [2]. Others couple the metric with other new gravitational degrees of freedom such as scalars [3,4], vectors [5,6], and tensors [7-10], which can arise in a variety of settings. Very few proposals consider the possibility that the gravitational action might be fundamentally nonlocal.

In some sense, the minimalist approach to modifying GR is to look at the effective action for gravity that arises from one-loop correction from gauge and matter fields [11-14]. While, at first sight, nonlocal interactions may seem to be a step too far, they seem to consistently crop up, and when they do, they can have far-reaching consequences. So, for example, the feted trace anomaly which was uncovered in the 1970s [15] played an instrumental role in the first model of inflation constructed by Starobinsky [16]. Surprisingly, it is this model that seems best to fit the current measurements of large scale structure coming out of the analysis of the Planck satellite data [17]. The role of the trace anomaly in the early Universe was also highlighted in Ref. [18], where it was shown that it could be used to generate large scale seed magnetic fields. While in detail, the proposal is problematic (it needs many fermion families and large gauge groups), it is still one of the frontrunners in explaining how these fields formed in the early Universe. Over the years, a number of proposals for the origin, and effect of, nonlocal terms in the gravitational action have been put forward [19-23].

More recently, Deser and Woodard [24] have embraced the possibility of nonlocal terms and proposed that the gravitational action could take the form

$$
S_{\mathrm{DW}}=-\frac{1}{16 \pi G} \int d^{4} \sqrt{g} R\left(1+f\left(\frac{R}{\square}\right)\right) .
$$

\footnotetext{
*p.ferreira1@physics.ox.ac.uk
}

maroto@ucm.es
Such an action has two appealing properties. For a start, the argument, $R / \square$, is dimensionless, which means that we expect $f(x)$ to be solely constructed in terms of dimensionless constants of order 1-in some sense. it is "natural." But, as the authors showed, the fact that it is divided by $\square$ means that the modification becomes large for large length scales and, more interestingly, for long time scales. Given that $R=0$ in pure radiation, they show that it is possible to construct a model which affects late time expansion yet is triggered by the radiation-matter transition [25]. A few authors have explored alternative local formulations constructed with additional scalar degrees of freedom [26-30].

It has also been argued that nonlocal terms in the gravitational action can be brought in to generate a graviton mass without the usual pathologies that arise in standard (but not modern) massive gravity [31]. The authors showed that such a modification did not lead to a van DamVeltman-Zakharov discontinuity, did not have ghosts [32], and, conveniently, led to dark-energy-like behavior at late time [33]. The phenomenon is akin to that of "degravitation," which was advocated in Ref. [34].

Nonlocal terms may also introduce modifications to the effective action for gauge fields [35-37]. Indeed, the complete effective action for gravity and gauge fields [11-14] clearly shows how an abundance of nonlocal terms of various forms and guises can emerge. It seems then that a sensible approach is to be ecumenical and study a wide range of nonlocal terms; a particularly simple setting is to find-as was done in Ref. [24] — how they effect the background expansion.

In this paper we extend the work of previous authors and look at general quadratic terms in the curvature tensor with a specific form of nonlocality (of the form " $1 / \square$ "). We determine the modified field equations and how the standard cosmological solutions is affected. We also look at how nonlocal corrections affect the photon dispersion relations, extending the work of Ref. [36]. In general we find that a growing mode generates a large instability, which rapidly dominates at late time. This means that great care must be had when studying these theories-such instabilities seem generic. Nevertheless, we argue that 
these terms should be studied in more detail and discuss possible limitations to our results.

\section{RECAP: ORIGIN OF NONLOCAL TERMS AND THE CASE OF $R / \square$}

The one-loop effective action obtained by integrating out quantum fields propagating on gravitational and/or gauge backgrounds is generically a nonlocal object which contains all the relevant information about the semiclassical evolution of the background fields. Different techniques to calculate it have been proposed in the literature, the most popular one being the so-called Schwinger-De Witt $[38,39]$ proper time representation. This method provides an asymptotic expansion in inverse powers of a mass parameter for which the coefficients themselves involve powers of curvatures and their derivatives. This expansion is covariant but local and can be typically applied when the variation scale of the background fields $L$ is much larger than the Compton wavelength of the quantum field which is integrated out, i.e., for $L \gg 1 / m$. This means that for massive fields, the effects of the new terms in the effective action are negligibly small on cosmological contexts. Even for massless fields, the Schwinger-De Witt expansion only contains information about the local divergences but not on the finite nonlocal terms, which could be relevant on large scales.

In order to overcome these difficulties, some techniques have been proposed such as the partial resummation of the Schwinger-De Witt expansion [40] obtaining part of the nonlocal structure. On the other hand, the so-called covariant perturbation theory [11-13], which is based on the fact that, in asymptotically flat backgrounds, the one-loop effective action is an analytic functional of the curvatures, allows us to expand the effective action in a nonlocal basis of curvature invariants up to any given order $N$. These methods show that for generic quadratic theories with inverse propagator

$$
H=g^{\mu \nu} \nabla_{\mu} \nabla_{\nu}+\left(\hat{P}-\frac{1}{6} R\right)
$$

(where $\hat{P}$ is the potential matrix of the field), the effective action contains generic nonlocal terms in the form

$$
\begin{aligned}
W_{\mathrm{NL}}= & \int d^{4} x \sqrt{g}\left(\sum_{i} \gamma_{i}\left(-\square_{2}\right) \mathcal{R}_{1} \mathcal{R}_{2}\right. \\
& \left.+\sum_{i} \Gamma_{i}\left(-\square_{1},-\square_{2},-\square_{3}\right) \mathcal{R}_{1} \mathcal{R}_{2} \mathcal{R}_{3}+\mathcal{O}\left(\mathcal{R}^{4}\right)\right),
\end{aligned}
$$

where the $i$ indices run over the dimension of the corresponding curvature basis. The generic curvatures $\mathcal{R}=\left(R_{\mu \nu}, \hat{\mathcal{R}}_{\mu \nu}, \hat{P}\right)$ correspond to either the Ricci tensor $R_{\mu \nu}$, the curvature associated to the arbitrary connection
$\hat{\mathcal{R}}_{\mu \nu}=\left[\nabla_{\mu}, \nabla_{\nu}\right]$, or $\hat{P}$. The nonlocal form factors $\gamma_{i}$ and $\Gamma_{i}$ typically contain $\log (\square)$ or inverse powers of $\square$ among other terms.

This kind of curvature expansion, despite the fact of being able to capture the essential nonlocal behavior and reproduce quantum anomalies from the effective action, are valid only when

$$
\nabla \nabla \mathcal{R} \gg \mathcal{R} \mathcal{R}
$$

which, as we will show below, is not necessarily satisfied in cosmological contexts. The possibility of constructing low-energy expansions, i.e., expansions valid in the opposite limit $\nabla \nabla \mathcal{R} \ll \mathcal{R} \mathcal{R}$, has been also explored [41,42], although the results in this case are very limited.

Nonlocal terms involving curvature tensors are also present in the nonlocal braneworld action of the RandallSundrum model considered in Refs. [43,44].

Deser and Woodard [24] have embraced the presence of these nonlocal terms to construct a nonlocal theory that leads to accelerated expansion. Their idea is based on a few simple, but key, observations. Consider Eq. (1), and take $R$ from a standard cosmology with either radiation or dust. We have that $R=0$ during the radiation dominated phase but transits to $R=4 /\left(3 t^{2}\right)$, where $t$ is physical time. If we take the simplest case of $f(x) \simeq x$ we need to solve for $\psi=R / \square$, i.e.,

$$
\square \psi=\ddot{\psi}+3 \frac{\dot{a}}{a} \dot{\psi}=R,
$$

with the appropriate boundary condition, we can easily integrate to find a logarithmically growing mode of the form $\ln \left(t / t_{\mathrm{eq}}\right)$, where $t_{\mathrm{eq}}$ is the time of equality. As a result, it is possible to get moderate but non-negligible effects on cosmological time scales. In this case, it is radiation-matter equality that can trigger the onset of what would be interpreted as dark energy domination. In its simplest incarnation, the $R / \square$ does not do the job, but the authors have shown that it is possible that a suitably retrofitted $f(x)$ can fit the observed expansion rate of the Universe [45]. Furthermore, it passes the usual astrophysical constraints on GR as well as being free of pathological degrees of freedom such as ghosts $[25,46]$.

While the proposal of Ref. [24] opens up an interesting avenue in gravitational physics, it makes sense to look at it in the wider context that motivates it. In particular, terms in $R / \square$ do not generally appear in isolation, as discussed above, and it is probable that other terms of a tensorial nature may contribute to, or even dominate, the gravitational dynamics. In the next section, we explore the properties of these terms, focusing on a nonlocal Ricci tensor coupling. 


\section{III. $R^{\mu \nu} \frac{1}{\square} R_{\mu \nu}$ TERM}

Let us consider the following nonlocal action for the gravitational sector:

$$
S_{G}=\int d^{4} x \sqrt{g}\left(-\frac{R}{16 \pi G}+M^{2} R^{\mu \nu} \frac{1}{\square} R_{\mu \nu}\right) .
$$

While this is a very specific choice, we believe it illustrates the key features that arise in nonlocal tensorial theories. Varying the action with respect to the metric tensor, we get

$$
\begin{aligned}
\delta S= & \int d^{4} x \sqrt{g} \delta g^{\alpha \beta}\left(\frac{1}{16 \pi G} G_{\alpha \beta}+M^{2}\left(-\frac{1}{2} g_{\alpha \beta} R^{\mu \nu} H_{\mu \nu}+2 R_{\beta}^{\mu} H_{\mu \alpha}+\square H_{\alpha \beta}+g_{\alpha \beta} \nabla_{\mu} \nabla_{\nu} H^{\mu \nu}-2 \nabla_{\mu} \nabla_{\beta} H_{\alpha}^{\mu}\right.\right. \\
& +\nabla_{\alpha} H^{\mu \nu} \nabla_{\beta} H_{\mu \nu}-\frac{1}{2} \nabla_{\lambda} H^{\mu \nu} \nabla^{\lambda} H_{\mu \nu} g_{\alpha \beta}-\frac{1}{2} H^{\mu \nu} \square H_{\mu \nu} g_{\alpha \beta}+2 H^{\mu \nu} \nabla_{\nu} \nabla_{\alpha} H_{\mu \beta}+2 \nabla_{\mu} H^{\mu \nu} \nabla_{\alpha} H_{\beta \nu} \\
& \left.\left.-2 \nabla_{\nu} H_{\beta}{ }^{\mu} \nabla_{\alpha} H^{\nu}{ }_{\mu}-2 H_{\beta}{ }^{\mu} \nabla_{\nu} \nabla_{\alpha} 4 H^{\nu}{ }_{\mu}\right)\right),
\end{aligned}
$$

where we have defined $H_{\alpha \beta}$ from $\square H_{\alpha \beta}=R_{\alpha \beta}$. Unlike previous works [47], the nonlocal term is not an analytic function of $\square$, and therefore in the variation procedure, we have followed the definitions in Ref. [48] in order to correctly enforce causality with two "formal" partial integrations. Thus, we have used

$$
\begin{aligned}
\int d^{4} x \sqrt{g} R^{\mu \nu} \delta\left(\frac{1}{\square} R_{\mu \nu}\right) & =\int d^{4} x \sqrt{g} R^{\mu \nu}\left(-\frac{1}{\square}(\delta \square)\left(\frac{1}{\square} R_{\mu \nu}\right)+\frac{1}{\square} \delta R_{\mu \nu}\right) \\
& =\int d^{4} x \sqrt{g}\left(\frac{1}{\square} R^{\mu \nu}\right)\left(-(\delta \square)\left(\frac{1}{\square} R_{\mu \nu}\right)+\delta R_{\mu \nu}\right) .
\end{aligned}
$$

We can rewrite the Einstein field equations in the suggestive form

$$
R_{\alpha \beta}-\frac{1}{2} g_{\alpha \beta} R=8 \pi G\left(T_{\alpha \beta}+T_{\alpha \beta}^{\mathrm{NL}}\right),
$$

where

$$
\begin{aligned}
T_{\alpha \beta}^{\mathrm{NL}}= & -2 M^{2}\left(-g_{\alpha \beta} R^{\mu \nu} H_{\mu \nu}+R_{\beta}^{\mu} H_{\mu \alpha}+R_{\alpha}^{\mu} H_{\mu \beta}+R_{\alpha \beta}+g_{\alpha \beta} \nabla_{\mu} \nabla_{\nu} H^{\mu \nu}-\nabla_{\mu} \nabla_{\beta} H_{\alpha}^{\mu}-\nabla_{\mu} \nabla_{\alpha} H_{\beta}^{\mu}\right. \\
& -\frac{1}{2} \nabla_{\lambda} H^{\mu \nu} \nabla^{\lambda} H_{\mu \nu} g_{\alpha \beta}+\nabla_{\alpha} H^{\mu \nu} \nabla_{\beta} H_{\mu \nu}+H^{\mu \nu} \nabla_{\nu} \nabla_{\alpha} H_{\mu \beta}+H^{\mu \nu} \nabla_{\nu} \nabla_{\beta} H_{\mu \alpha}+\nabla_{\mu} H^{\mu \nu} \nabla_{\alpha} H_{\beta \nu} \\
& \left.+\nabla_{\mu} H^{\mu \nu} \nabla_{\beta} H_{\alpha \nu}-\nabla_{\nu} H_{\beta}{ }^{\mu} \nabla_{\alpha} H^{\nu}{ }_{\mu}-\nabla_{\nu} H_{\alpha}{ }^{\mu} \nabla_{\beta} H_{\mu}^{\nu}{ }_{\mu}-H_{\beta}{ }^{\mu} \nabla_{\nu} \nabla_{\alpha} H_{\mu}^{\nu}-H_{\alpha}{ }^{\mu} \nabla_{\nu} \nabla_{\beta} H^{\nu}{ }_{\mu}\right),
\end{aligned}
$$

where $T_{\mathrm{NL} ; \mu}^{\mu \nu}=0$ has been checked explicitly on the solutions.

Let us now try and solve $H_{\alpha \beta}$. We will mimic Ref. [24] and assume that the nonlocal terms are a perturbation on a general relativistic background. We will be working with the conformal metric $g_{\alpha \beta}=a^{2}(\eta)\left(d \eta^{2}-d \vec{r}^{2}\right)$ and, given the symmetries of the problem, we assume

$$
H_{\nu}^{\mu}=\operatorname{diag}\left(H_{t},-H_{s},-H_{s},-H_{s}\right),
$$

which satisfy

$$
\begin{aligned}
& H_{t}^{\prime \prime}+2 \mathcal{H} H_{t}^{\prime}-6 \mathcal{H}^{2}\left(H_{t}+H_{s}\right)=3\left(\mathcal{H}^{2}-\frac{a^{\prime \prime}}{a}\right) \\
& H_{s}^{\prime \prime}+2 \mathcal{H} H_{s}^{\prime}-2 \mathcal{H}^{2}\left(H_{t}+H_{s}\right)=\left(\mathcal{H}^{2}+\frac{a^{\prime \prime}}{a}\right) .
\end{aligned}
$$

The system can be diagonalized if we define two new functions, $S=H_{t}+H_{s}$ and $D=H_{t}-3 H_{s}$, and we obtain

$$
\begin{gathered}
S^{\prime \prime}+2 \mathcal{H} S^{\prime}-8 \mathcal{H}^{2} S=4 \mathcal{H}^{2}-2 \frac{a^{\prime \prime}}{a} \\
D^{\prime \prime}+2 \mathcal{H} D^{\prime}=-6 \frac{a^{\prime \prime}}{a} .
\end{gathered}
$$

Equations (4) and (5) are equivalent to those found in Ref. [49] and present a key feature of tensor nonlocalities. From Eq. (3) we see that the d'Alembertian of a scalar field consists solely of derivative terms [recall that $\square \psi=$ $\left.\frac{1}{\sqrt{g}} \partial_{\mu}\left(\sqrt{g} g^{\mu \nu} \partial_{\nu} \psi\right)\right]$. This is not the case for tensor fields where there will be nonderivative terms, schematically of the form $\sim \Gamma \Gamma H$, where $\Gamma$ is the connection coefficient. Depending on the sign of these terms, they lead to an 
oscillatory or, as we see in Eq. (4), a growing mode. As we shall see, this means that the tensor nonlocalities will dominate the dynamics. Notice also that the $D$ mode which corresponds to the trace of $H^{\mu}{ }_{\nu}$ does not exhibit growing solutions of the homogeneous equation.

To illustrate the point we have just made, we now solve for $S$ and $D$ for particular choices of backgrounds. At late times we can assume matter domination with $a \propto \eta^{2}$, and we then find

$$
S=C_{+} \eta^{p_{+}}+C_{-} \eta^{p_{-}}-\frac{3}{8} \quad D=\frac{C_{1}}{\eta^{3}}-4 \ln \eta+C_{2},
$$

where $p_{ \pm}=-\frac{3}{2} \pm \frac{\sqrt{137}}{2}$. If the Universe is perfectly matter dominated throughout, we would have that the boundary conditions could be such that $C_{+}=0$, and again we would have, at most, a logarithmic growth. But this is not the case; we have to match onto a nonzero solution emerging from the radiation era (unlike for the case of the $R / \square$ term), which will generally stimulate a $C_{+}$term. This dominant, growing, mode evolves as $S \propto \eta^{4.35} \propto a^{2.18}$ and leads to a $T_{\alpha \beta}^{\mathrm{NL}}$ such that $\rho_{\mathrm{NL}} \propto \eta^{2 p_{+}-6}$, which corresponds to a fluid with an equation of state $w_{\mathrm{NL}}=-1.451$. That is, the tensor nonlocality is responsible for a dominant growing modification which rapidly drowns out any slow, desirable, logarithmic modification.

That there is a nonzero solution emerging from the radiation era is easy to show. Choosing $a \propto \eta$ one has

$$
S=C_{+} \eta^{p_{+}}+C_{-} \eta^{p_{-}}-\frac{1}{2} \quad D=C_{1}+\frac{C_{2}}{\eta},
$$

where $p_{ \pm}=-\frac{1}{2} \pm \frac{\sqrt{33}}{2}$. The best one could hope for is a constant solution, but any residual matter or a transition in the effective degrees of freedom will perturb the evolution away and seed a growing mode such that $S \propto \eta^{2.37} \propto a^{2.37}$. We then have $\rho_{\mathrm{NL}} \propto \eta^{2 p_{+}-4}$ so that $w_{\mathrm{NL}}=-1.248$. Finally, for completeness, if we push back to a primordial inflationary regime, we have $a \propto-1 / \eta$, which leads to

$S=C_{+} \eta^{p_{+}}+C_{-} \eta^{p_{-}} \quad D=C_{1} \eta^{3}+4 \ln (-\eta)+C_{2}$,

where $p_{ \pm}=\frac{3}{2} \mp \frac{\sqrt{41}}{2}$ and $p_{+}$corresponds to the growing mode. Again, this is for pure de Sitter, and any small deviation will trigger a growing mode such that $S \propto$ $(-\eta)^{-1.70} \propto a^{1.70}$ so that $\rho_{\mathrm{NL}} \propto(-\eta)^{2 p_{+}}$and therefore $w_{\mathrm{NL}}=-2.134$

In general we find that

$$
\rho_{\mathrm{NL}} \propto \rho_{\mathrm{bk}}|\eta|^{2 p_{+}},
$$

with $\rho_{\mathrm{bk}}$ the background energy density driving the expansion. This means that, very rapidly, the growing mode in the tensor nonlocalities comes into play, and the modified dynamics dominates the expansion rate of the Universe, unless the scale $M$ is extremely small. Furthermore, the quadratic terms, which have been generally discarded in the past $[23,33,49,50]$, cannot be ignored and dominate the behavior of $T_{\alpha \beta}^{\mathrm{NL}}$.

We have focused on one particular nonlocal, tensorial, term in the gravitational action, yet, from the discussion in the previous section and full action presented in Refs. [11-13], we expect other possible terms. So, for example, we might consider nonlocal terms involving the full Riemann tensor of the form

$$
\frac{R^{\mu \nu}{ }_{\rho \sigma}}{\square}=H_{\rho \sigma}^{\mu \nu} .
$$

But these can be rewritten in terms of the Ricci tensor and scalar; in a Robertson-Walker background, the Weyl tensor identically vanishes, so that

$$
R_{\rho \sigma}^{\mu \nu}=g_{[\rho}^{\mu} R_{\sigma]}^{\nu}-g_{[\rho}^{\nu} R_{\sigma]}^{\mu}-\frac{1}{3} g_{[\rho}^{\mu} g_{\sigma]}^{\nu} R,
$$

and therefore, using the fact that the metric tensor commutes with $1 / \square$, we have:

$$
R_{\mu \nu}{ }^{\rho \sigma} \frac{1}{\square} R_{\rho \sigma}^{\mu \nu}=-\frac{1}{3} R \frac{1}{\square} R+2 R_{\mu \nu} \frac{1}{\square} R^{\mu \nu} .
$$

Hence, the results that we have found for the $R_{\mu \nu} / \square$ case can be easily applied here. Notice also that the same kind of arguments can be applied to terms like $G_{\mu \nu} \frac{1}{\square} R^{\mu \nu}$ considered in Ref. [50].

The argument made above is general: anything with an index may lead to a growing mode. So, for example, if we consider a nonlocal vector term of the form

$$
\frac{V^{\mu}}{\square}=H^{\mu}
$$

and restrict ourselves to homogeneity and isotropy such that $H^{\mu}=\left(H^{0}(\eta), 0,0,0\right)$, then we have

$$
a^{-4}\left(H^{0 \prime \prime}-\left(3 \mathcal{H}^{2}+\frac{a^{\prime \prime}}{a}\right) H^{0}\right)=V^{0} .
$$

Once again, we find power law solutions for the homogeneous equation

$$
H^{0}=C_{+} \eta^{p_{+}}+C_{-} \eta^{p_{-}},
$$

with $p_{ \pm}=(1 \pm \sqrt{13}) / 2$ during the radiation era, $p_{ \pm}=$ $(1 \pm \sqrt{97}) / 2$ during the matter era, and $p_{ \pm}=(1 \pm$ $\sqrt{21}) / 2$ during the inflationary era.

\section{NONLOCAL PHOTON}

In the previous section, we focused on the gravitational dynamics and how it is affected by nonlocal terms. But a crucial component of cosmology is how we observe the expansion rate-we do this with photons via luminosity and angular diameter distances. As shown before, nonlocal terms involving Ricci and gauge curvatures are also expected when calculating the one-loop effective action, and so it makes sense to focus on an Abelian gauge theory in a 
curved space-time and how it may be affected by the simplest tensor nonlocality:

$$
\begin{aligned}
S & =\int d^{4} x \sqrt{g}\left(-\frac{1}{4} F^{\mu \nu} F_{\mu \nu}+\alpha \frac{R^{\alpha}{ }_{\beta}}{\square} F_{\mu \alpha} F^{\mu \beta}\right) \\
& =\int d^{4} x \sqrt{g}\left(-\frac{1}{4} F^{\mu \nu} F_{\mu \nu}+\alpha H^{\alpha}{ }_{\beta} F_{\mu \alpha} F^{\mu \beta}\right) .
\end{aligned}
$$

In such a theory, the modified Maxwell equations are

$$
\frac{1}{\sqrt{g}} \partial_{\mu}\left(\sqrt{g} F^{\mu \nu}\right)-\frac{2 \alpha}{\sqrt{g}} \partial_{\mu}\left(\sqrt{g}\left(H_{\alpha}{ }^{\nu} F^{\mu \alpha}-H_{\alpha}{ }^{\mu} F^{\nu \alpha}\right)\right)=0 .
$$

Let us now focus on the temporal component of the Faraday tensor (i.e., setting $\nu=0$ ). We then have

$$
\nabla_{\mu} F^{\mu 0}-2 \alpha \nabla_{\mu}\left(H_{\alpha}{ }^{0} F^{\mu \alpha}-H_{\alpha}{ }^{\mu} F^{0 \alpha}\right)=0,
$$

which in a conformal space-time takes the form

$$
-\frac{1}{a^{4}} \partial_{i} F_{i 0}+\frac{2 \alpha}{a^{4}} \partial_{i}\left(H_{0}{ }^{0} F_{i 0}-H_{j}{ }^{i} F_{0 j}\right)=0
$$

and greatly simplifies to

$$
\left[1-2 \alpha\left(H_{t}-H_{s}\right)\right] \partial_{i} F_{i 0}=0,
$$

where $H_{t}$ and $H_{s}$ were defined and calculated in the previous section. With the Coulomb gauge, $\vec{\nabla} \cdot \vec{A}=0$, this leads to $\nabla^{2} A_{0}=0$, and, in the absence of sources, we can choose $A_{0}=0$.

Let us now consider a wave of the form $A_{\mu}=$ $\left(0, A_{i}(\eta) e^{i \vec{k} \vec{x}}\right)$ such that $\vec{k} \cdot \vec{A}=0$. The spatial part of the Maxwell equations is now

$$
\nabla_{\mu} F^{\mu i}-2 \alpha \nabla_{\mu}\left(H_{\alpha}{ }^{i} F^{\mu \alpha}-H_{\alpha}{ }^{\mu} F^{i \alpha}\right)=0,
$$

which leads to

$$
A_{i}^{\prime \prime}+\frac{1+4 \alpha H_{s}}{1+2 \alpha\left(H_{s}-H_{t}\right)} \vec{k}^{2} A_{i}+\frac{2 \alpha\left(H_{s}^{\prime}-H_{t}^{\prime}\right)}{1+2 \alpha\left(H_{s}-H_{t}\right)} A_{i}^{\prime}=0 .
$$

We can explore the properties of this equation in light of what we learned in the previous section. Taking only the growing mode, we will have that the $S$ part of $H_{s}$ and $H_{t}$ will dominate, leading to

$$
A_{i}^{\prime \prime}-\frac{\alpha S^{\prime}}{1-\alpha S} A_{i}^{\prime}+\frac{1+\alpha S}{1-\alpha S} \vec{k}^{2} A_{i}=0 .
$$

Furthermore, asymptotically $|S| \gg 1$, so that

$$
A_{i}^{\prime \prime}+\frac{S^{\prime}}{S} A_{i}^{\prime}-k^{2} A_{i}=0 .
$$

If, as we saw in the last section, $S \propto \eta^{p}$, we have that $A_{i} \propto$ $\eta^{\frac{1-p}{2}} I_{q}(|k| \eta)$, where $q^{2}=(p-1) p / 4$ and $I_{q}(x)$ is a modified Bessel function; i.e., asymptotically the solution diverges exponentially.
To understand the structural effects of the nonlocal term, it is useful to solve Eq. (6) using the geometric optics approximation [51] in which the electromagnetic field $A_{\mu}$ is assumed to behave as a fast evolving phase times a slowly evolving amplitude, i.e.,

$$
A_{\mu}=\left(a_{\mu}+\epsilon b_{\mu}+\cdots\right) e^{i \theta / \epsilon},
$$

where the $\epsilon$ parameter controls how rapidly each term evolves. Defining $k_{\mu}=\partial_{\mu} \theta$ and substituting the expansion in Eq. (6) keeping only the leading terms of order $1 / \epsilon^{2}$, we obtain

$$
\begin{aligned}
& -k^{2} a^{\nu}+2 \alpha H_{\alpha}{ }^{\nu} k^{2} a^{\alpha}-2 \alpha H_{\alpha}{ }^{\mu}\left(k_{\mu} k^{\nu} a^{\alpha}-k_{\mu} k^{\alpha} a^{\nu}\right) \\
& \quad=0
\end{aligned}
$$

where $k^{2}=k_{\mu} k^{\mu}$ and we have used the Lorenz gauge condition $\nabla_{\mu} A^{\mu}=0$, which implies, to leading order $k_{\mu} a^{\mu}=0$.

As above we find

$$
\left[1-2 \alpha\left(H_{t}-H_{s}\right)\right] k^{2} a^{0}=0
$$

and

$$
\begin{aligned}
& -k^{2} a^{i}-2 \alpha H_{s} k^{2} a^{i}+2 \alpha H_{t} k_{0} k^{0} a^{i}-2 \alpha H_{s} k_{j} k^{j} a^{i} \\
& -2 \alpha H_{t} k_{0} a^{0} k^{i}+2 \alpha H_{s} k_{j} a^{j} k^{i}=0,
\end{aligned}
$$

for which the nontrivial solutions imply $a^{0}=0$ so that $a^{i} k_{i}=0$ and therefore

$$
k_{0} k^{0}\left(1+2 \alpha\left(H_{s}-H_{t}\right)\right)+k_{j} k^{j}\left(1+4 \alpha H_{s}\right)=0 ;
$$

i.e., keeping only the growing mode, the modified dispersion relation reads

$$
\omega^{2}=\frac{1+\alpha S}{1-\alpha S} \vec{k}^{2},
$$

with $\omega^{2}=k_{0}^{2}$ and $\vec{k}^{2}=k_{i} k_{i}$, which agrees with the previous result.

For the $1 / \epsilon$ terms, we get

$$
\begin{aligned}
- & k^{2} b^{\nu}+2 H_{\alpha}{ }^{\nu} k^{2} b^{\alpha}-2 \alpha H_{\alpha}{ }^{\mu}\left(k_{\mu} k^{\nu} b^{\alpha}-k_{\mu} k^{\alpha} b^{\nu}\right)+2 i k^{\alpha} a_{; \alpha}^{\nu} \\
& +i k_{; \alpha}^{\alpha} a^{\nu}+2 i \alpha H_{\alpha}{ }^{\nu}{ }_{; \mu}\left(k^{\mu} a^{\alpha}-k^{\alpha} a^{\mu}\right)-4 i \alpha H_{\alpha}{ }^{\nu} k^{\beta} a^{\alpha}{ }_{; \beta} \\
& -2 i \alpha H_{\alpha}{ }^{\nu} k_{; \beta}^{\beta} a^{\alpha}+2 i \alpha H_{\alpha}{ }^{\mu}{ }_{; \mu}\left(k^{\nu} a^{\alpha}-k^{\alpha} a^{\nu}\right) \\
& +2 i \alpha H_{\alpha}{ }^{\mu}\left(k_{; \mu}^{\nu} a^{\alpha}-k_{; \mu}^{\alpha} a^{\nu}\right) \\
& +2 i \alpha H_{\alpha}{ }^{\mu}\left(k^{\nu} a_{; \mu}^{\alpha}-k^{\alpha} a_{; \mu}^{\nu}\right)=0 .
\end{aligned}
$$

Using the order $1 / \epsilon^{2}$ equations (7), it is possible to cancel the terms containing $b^{\mu}$ in the first line so that we are left with an equation for $a^{\mu}$ which describes the propagation of the amplitude and polarization of the field.

Thus, in the Robertson-Walker background, assuming also homogeneity of $a_{\nu}$ and $k_{\nu}$, i.e., $\partial_{i} a_{\nu}=\partial_{i} k_{\nu}=0$, and using the expression for $H^{\mu}{ }_{\nu}$, we get

$$
\partial_{0}\left(k^{0} A^{2}\left(1+2 \alpha\left(H_{s}-H_{t}\right)\right)\right)=0,
$$


where we have written $a_{\nu}=A f_{\nu}$, here $A$ is the wave amplitude and $f_{\nu}$ a unit polarization vector with $f^{0}=0$. Using the previous equation, we can also obtain

$$
\left(1+2 \alpha\left(H_{s}-H_{t}\right)\right) \partial_{0} f_{i}=0 ;
$$

i.e., the polarization vector remains constant in the evolution, in agreement with the previous results. We also find in Eq. (8) a modification of the so-called area law of ordinary electromagnetism, which is directly related to photon number conservation [51]. However, in this case, the standard interpretation in terms of photons is not appropriate at late times due to the fact that the electromagnetic modes will grow exponentially because of the tachyon instability.

\section{DISCUSSION}

In this paper, and following on from Ref. [24], we have explored the role of nonlocal corrections to the dynamics of the Universe. While in Ref. [24], the authors have attempted to flesh out a working model, we have opted to be agnostic and have chosen the one-loop effective action from Refs. [11-14] as our starting point. As such we have had to address the importance of tensor nonlocalities and have found that, in general, they dominate the evolution of the Universe at late times. We have, of course, taken a simplified, perturbative approach and shown that, because of a very rapidly growing solution, quadratic terms in the expansion become important. The result suggests that higher and higher curvature terms would become more and more important, thus signaling the breakdown of this kind of nonlocal curvature expansion in the cosmological context.

A similar result has been obtained in the case of the nonlocal contributions to the electromagnetic action. In the simplest case with tensor nonlocalities, the growing solution induces a tachyon instability in the electromagnetic field at late times, which again signals the inadequacy of this kind of truncated nonlocal curvature expansions in cosmology. Although in this work we have concentrated on two particular examples, these kinds of instabilities seem to be generic in the case of tensor nonlocalities, in contrast with the scalar cases in which they are absent.

Clearly a few things need to be done. For a start it would make sense to undertake a full, self-consistent, analysis of the modified field equations to see if the growth could be suppressed in certain particular scenarios in which this kind of effective action could be applicable. It is unlikely that this is the case, but possible and should be checked. Furthermore, there are other possible nonlocal operators that one might consider. One example would be $\square+m^{2}$, where the mass term might regularize the growing mode. Another example would be the operator that arises with the conformal anomaly, $\square^{2}+2 \nabla_{\mu}\left[R^{\mu \nu}-\frac{1}{3} g^{\mu \nu}\right] \nabla_{\mu}$. It is conceivable that such operators might lead to somewhat different behavior.

More ambitiously, one needs to come up with a more complete effective action for gravity, including more than just the quadratic or cubic terms in curvatures that have been considered in this analysis. Going beyond the regime of small enough curvature or large derivatives defined by Eq. (2) would shed new light on the role of quantum effects on the late-time cosmological evolution.

\section{ACKNOWLEDGMENTS}

We are extremely grateful to R. Woodard for his advice and guidance and to Tessa Baker, Joe Conlon, and Johannes Noller for discussions. P. G.F. acknowledges support from Leverhulme, STFC, BIPAC, and the Oxford Martin School and the hospitality of the Higgs Centre in Edinburgh while this paper was being completed. A.L.M. acknowledges support from MICINN (Spain) Projects No. FIS2011-23000 and Consolider-Ingenio MULTIDARK No. CSD2009-00064, the Salvador de Madariaga program, and the hospitality of Oxford BIPAC.
[1] T. Clifton, P. G. Ferreira, A. Padilla, and C. Skordis, Phys. Rep. 513, 1 (2012).

[2] T. P. Sotiriou and V. Faraoni, Rev. Mod. Phys. 82, 451 (2010).

[3] C. Brans and R. Dicke, Phys. Rev. 124, 925 (1961).

[4] G. W. Horndeski, Int. J. Theor. Phys. 10, 363 (1974).

[5] T. G. Zlosnik, P. G. Ferreira, and G. D. Starkman, Phys. Rev. D 75, 044017 (2007).

[6] J. Beltrán Jiménez and A. L. Maroto, Phys. Rev. D 80, 063512 (2009).

[7] M. Bañados, P. G. Ferreira, and C. Skordis, Phys. Rev. D 79, 063511 (2009).

[8] M. Bañados and P. G. Ferreira, Phys. Rev. Lett. 105, 011101 (2010).
[9] C. de Rham, G. Gabadadze, and A. J. Tolley, Phys. Rev. Lett. 106, 231101 (2011).

[10] S. F. Hassan and R. A. Rosen, J. High Energy Phys. 02 (2012) 126.

[11] A. Barvinsky and G. Vilkovisky, Nucl. Phys. B282, 163 (1987).

[12] A. Barvinsky and G. Vilkovisky, Nucl. Phys. B333, 471 (1990).

[13] A. Barvinsky and G. Vilkovisky, Nucl. Phys. B333, 512 (1990).

[14] A. Barvinsky, Y. Gusev, V. Zhytnikov, and G. Vilkovisky, Classical Quantum Gravity 12, 2157 (1995).

[15] D. Capper and M. Duff, Nuovo Cimento A 23, 173 (1974).

[16] A. A. Starobinsky, Phys. Lett. 91B, 99 (1980). 
[17] P. Ade et al. (Planck Collaboration), arXiv:1303.5076.

[18] A. Dolgov, Phys. Rev. D 48, 2499 (1993).

[19] C. Wetterich, Nucl. Phys. B302, 668 (1988).

[20] T. Banks, Nucl. Phys. B309, 493 (1988).

[21] N. Tsamis and R. Woodard, Ann. Phys. (N.Y.) 267, 145 (1998).

[22] D. Espriu, T. Multamaki, and E. C. Vagenas, Phys. Lett. B 628, 197 (2005).

[23] H. W. Hamber and R. M. Williams, Phys. Rev. D 72, 044026 (2005).

[24] S. Deser and R. Woodard, Phys. Rev. Lett. 99, 111301 (2007).

[25] S. Deser and R. Woodard, arXiv:1307.6639.

[26] S. Nojiri and S.D. Odintsov, Phys. Lett. B 659, 821 (2008).

[27] S. Jhingan, S. Nojiri, S.D. Odintsov, M. Sami, I. Thongkool, and S. Zerbini, Phys. Lett. B 663, 424 (2008).

[28] T. Koivisto, Phys. Rev. D 77, 123513 (2008).

[29] S. Nojiri, S. D. Odintsov, M. Sasaki, and Y.-1. Zhang, Phys. Lett. B 696, 278 (2011).

[30] E. Elizalde, E. O. Pozdeeva, S. Y. Vernov, and Y.-1. Zhang, J. Cosmol. Astropart. Phys. 07 (2013) 034.

[31] M. Jaccard, M. Maggiore, and E. Mitsou, Phys. Rev. D 88, 044033 (2013).

[32] T. Biswas, E. Gerwick, T. Koivisto, and A. Mazumdar, Phys. Rev. Lett. 108, 031101 (2012).

[33] M. Maggiore, arXiv:1307.3898.
[34] N. Arkani-Hamed, S. Dimopoulos, G. Dvali, and G. Gabadadze, arXiv:hep-th/0209227.

[35] I. Drummond and S. Hathrell, Phys. Rev. D 22, 343 (1980).

[36] G. Shore, Nucl. Phys. B460, 379 (1996).

[37] R. Riegert, Phys. Lett. 134B, 56 (1984).

[38] J. S. Schwinger, Phys. Rev. 82, 664 (1951).

[39] B. DeWitt, Dynamical Theory of Groups and Fields (Gordon and Breach, New York, 1965).

[40] I. Avramidi, Nucl. Phys. B355, 712 (1991).

[41] I. Avramidi, J. Math. Phys. (N.Y.) 37, 374 (1996).

[42] I. G. Avramidi, Adv. Theor. Math. Phys. 14, 309 (2010).

[43] A. Barvinsky, Phys. Rev. D 65, 062003 (2002).

[44] A. O. Barvinsky, A. Y. Kamenshchik, A. Rathke, and C. Kiefer, Phys. Rev. D 67, 023513 (2003).

[45] C. Deffayet and R. Woodard, J. Cosmol. Astropart. Phys. 08 (2009) 023.

[46] S. Park and S. Dodelson, Phys. Rev. D 87, 024003 (2013).

[47] A. S. Koshelev, Classical Quantum Gravity 30, 155001 (2013).

[48] M. Soussa and R. P. Woodard, Classical Quantum Gravity 20, 2737 (2003).

[49] L. Modesto and S. Tsujikawa, Phys. Lett. B 727, 48 (2013).

[50] A. Barvinsky, Phys. Lett. B 572, 109 (2003).

[51] C. Misner, K. Thorne, and J. Wheeler, Gravitation (Freeman, New York, 1973). 